\title{
New Trend Proposal in Optimization Techniques Application for Mobile Network, Analysis and Signal Processing
}

\author{
Chafaa HAMROUNI ${ }^{1,2 *}$
}

\begin{abstract}
Used optimization techniques as solution for mobile network have been implemented as a reference systems for various applications against fading and signals perturbation, in addition each transition to 5th generation telecommunication standards require a deep studies in order to park an applied instantaneous process. The paper describes a preliminary planning and a careful preparation to update both subscriber radio access network as well as data transmission network this approach conducts to make network resource updates invisible for customers and with minimal costs for mobile operators basically in terms of delay. In addition, network operators transit to mobile networks, multimedia services efficient delivery are considered the challenging application and the most promising for mobile network operators today, this work conduct to optimize video consumption of mobile users which are exponentially increasing. The interference is a complex phenomenon in mobile radio telecommunication system, and a mobile phone can be a source of interference to another one. Actual advances in technology necessitate the need for the complicated software solution that can take several unexpected phenomena in consideration to rise to a level higher than ever. The capability needs today require the use of Drive test which is used to take the performance of network in the field by using a special software called TEMS investigation, it have been implemented as standalone systems for various applications. The paper focuses on considering as the best technical for optimization of mobile networks, analysis and processing of signal, a Drive Test is the method used to take the performance of network in the field by using a special software called TEMS investigation. Most used in the world, this software is reputed to detect and analyze many problems of mobile network between the mobile phone and the transmitter: BTS in case of GSM and Node B for UMTS. An example of that is interference in radio communication. It exists permanently and it degrades considerably the quality of received signal when it exceeds certain levels.
\end{abstract}

Key Words: Drive Test, TEMS Investigation, GSM.

\section{INTRODUCTION}

In practice, the optimized method used to verify and measure mobile network parameters performance degrees, depends on tests types and precision. We propose a specific software, used often with car drive, that we call Drive test. To save a minimum performance level and Quality of services (Qos) [1], and each mobile Telecommunication services provider are asked to validate a minimum Drive tests [2] evaluation results. These tests control and granite the efficiencies of the mobile network to be more competitive and performer over time. submit the final version. The instructions are designed for the preparation of a camera-ready and accepted paper in MS Word and should be read carefully.

A Drive Test operates in air interface, and operates between Mobile Station and the transmitter, that means the
BTS or Node B. TEMS [3] license is important, and the benefits by using the technology are interesting. We propose Drive Test in order to optimize a mobile network, it is a new method of coverage measures, capacity and QoS of the mobile network [4]. As the name suggest, we use a motor vehicle outfitted an equipment for detecting and recording wide variety of the physical and virtual parameters [5] of mobile cellular service to evaluate in geographical area. We apply the method for $2 \mathrm{G}, 2.5 \mathrm{G}, 3 \mathrm{G}$, 4G, WiMAX, WLL etc. We propose three steps to accomplish the tests: firstly, planning step which deals with determination of where and when the drive will take place, at night or during the day. We are asked to have several information of the site [6] like such Site master database that is antenna parameter, downloaded digital maps of site (from google earth, frequency plan, Drive test equipment, etc. Secondly, implementation step that takes field

\footnotetext{
Manuscript received July 01, 2020; Revised August 03; Accepted August 29, 2020. (ID No. JMIS-20M-07-020)

Corresponding Author $(*)$ : Chafaa Hamrouni, Taif Umniversity, Khurma University College, Dep. of Computer Sciences, Kingdom of Saudia Aribia, ${ }^{1} \mathrm{cmhamrouni} @$ tu.edu.sa

${ }^{2}$ REGIM Lab. ENIS, University of Sfax, Tunisia, 00966546492338, chafa.hamrouni.tn@ ieee.org
} 
measurement. Thirdly, the optimization step which develop and implement an optimized solution for the problem detected during the implementation step [7].

\section{ADOPTED METHODOLOGY}

During tests step, we are allowed to select materials depending on site characteristics. We propose the following common materials list composed by a laptop, data collecting software such TEMS, a mobile phone, a GPS and cables. In tests phase, the mobile takes the information about the signal located on each position whose latitude and longitude are given by GPS. All data are collected by the laptop into logfiles for later analysis [8]. There are different Drive test type as well many way to classify the Drive test, and according to this purpose, the main types of Drive tests are performance analysis, which is used to measure and verify the performance of mobile network. The integration of new sites and change parameters of existing site is adopted coverage cell area evaluation. An antenna redesign used to define cell/sites coverage. Benchmarking is to compare the performance of different mobile devices [9], such between i-phone and Samsung phone and/or different mobile networks between mobile phone services providers.

We determine Drive test parameters based on Bit Error Rate (BER) [10] and RxQual representing the percentage of bits errors decoded by the mobile phone in 465 bits. It indicates also directly the quality of received signal called RxQual. For data collecting, the mobile and the GPS are connected to PC, Drive Tests operation can be started, and then the following data are collected by measurement, which are:

- Signal intensity

- Interference

- Dropped calls

- Anomalous events

- Call statistics

- Service level statistics

- Handover information

- Neighboring cell information

The experimental results are presented in Table 1. Table 1. BER to RxQual.

\begin{tabular}{|c|l|}
\hline RxQual & \multicolumn{1}{|c|}{ Bit Error Rate } \\
\hline 1 & $\mathrm{BER} \leq 0.2 \%$ \\
\hline 2 & $0.2 \% \leq \mathrm{BER} \leq 0.4 \%$ \\
\hline 3 & $0.4 \% \leq \mathrm{BER} \leq 0.8 \%$ \\
\hline 4 & $0.8 \% \leq \mathrm{BER} \leq 1.6 \%$ \\
\hline 5 & $1.6 \% \leq \mathrm{BER} \leq 3.2 \% \%$ \\
\hline 6 & $3.2 \% \leq \mathrm{BER} \leq 6.4 \%$ \\
\hline 7 & $6.4 \% \leq \mathrm{BER}$ \\
\hline
\end{tabular}

The carrier-over-interference is the ratio between signal strength of current serving cell and undesired signal. RxLev Full is measured when DTX is off and Sub otherwise. Tow ranges are presented Rang1 defined in interval 12 to 32 excellent 5 to 9 poor, and Rang2 representing the interval from 9 to 12 average -5 to 5 worst. The RX-Level or Rxlev is the power level, in $\mathrm{dBm}$, corresponding to average received signal down-link. Rxlev Full is measured when discontinuous transmission (DTX) is on and Sub otherwise. The frame erasure rate (FER) indicates fading interference which affect voice quality. It is percentage of errors in block of bit after verified by CRC (Cyclic Redundancy Check). The speech quality index (SQI) is the perceived speech quality estimate. Besides a bits errors, there are another parameters like handover events and frame erasure distributions define the quality of speech. Background noise, echo problems and other problems affect also listening during a call. And the Ranges are defined in Rande. 1 in interval from 25 to 31 excellent 5 to 14 average, 14 to 25 good -5 to 5 worst. For the Code division multiple access parameters (CDMA). Experimentally, we define four parameters respectively: Received Signal Code Power or RSCP: Like RxLev in the GSM, Carrier Energy to Interference or Ec/Io: the ratio of received pilot energy, eEc, to total received energy or the total power special density, Io. Value less than $-13 \mathrm{~dB}$ indicates bad terrain, interference and increased number of users, Tx Power: determines how the transmitting power of the mobile station. It will vary in range -50 to 50 . Value less than -20 indicates drop call, call block., Carrier to Interference: defines interference between two cells using the same frequency. Value more than $12 \mathrm{~dB}$ indicates the interference must be solved. item Signal to Noise Ratio: should be less than 6, I suggest visiting a linked in slide share website, Drive Test Parameters [11]

The adopted Drive test modes for a determined specific target, we define three modes: firstly, the call mode, where expert makes a short call to test its establishment. Experimentally, for handover events, the call must be long. Secondary, the IDLE mode, where mobile [12] is switch on and does not make a call. It is connected to the base station transmitting some mode parameters and data of location., and thirdly, Scan Mode Unlike Call and IDLE mode [13], this mode is one of TEMS features [14]. The Drive tester scans all/part of frequencies to detect and solve many problems of mobile network specially interference. The problems identified by using drive test and proposed solutions can be determined by:

- Coverage problems [15]: Increased number of users mainly causes two problems: poor coverage and low signal level.

- Lack of dominant server : This is happening when the cell 
phone [16] is located on cell borders and there are any best server to keep the call.

- Sudden decrease on signal level [17] : The mainly cause of this problem is an excessive number of handover on place. The other cause is presented if the mobile phone has a good signal level form [18] a far BTS in specific area and the signal is suddenly decrease when the mobile left and connect to the normal BTS serving this area. A tunnel and some of construction material cause also this situation. Cell over shooting [19]: The cell serves beyond its specific area. It often causes the interference to neighbor cell and the call drop.

- Missing Neighbor Relation [20]: The handover does not take place between two cells because they aren't assigned as neighbors. This list is exhaustive. Concerning the problem of low signal strength [21], the best recommend dation is to create a new site. Sometimes, it's very expensive and there is not high demand on traffic so the second thing to do is use the repeater. About the cell overshooting problem, adjusting antenna parameters such as: its azimuth, its down tilt its power, is the best thing to do to restrict its coverage [22]. Once identified the two cells causing problem to handover, we add the neighbor relation and make sure it really takes place. We verify the quality of network in the field. It allows to resolve many complaints of subscriber, detect the actual cause of the problem [23] and proceed to improvement. The test mobile system is a software for a Drive test, it can be installed on Windows XP, Windows Vista, Windows 7 and Windows 8. And it supports several operating systems such Android, Symbian, iOS, Windows Phone. According to its official website [24], nowadays, Ascom gives updates and services only to eight TEMS products and it has stopped production for the others. it is installed on volunteer subscriber' phone and collects its network data and sends the latest to Ascom servers. After, the operator and Ascom can both accesses, analyze on it. The software supports 2G, 3G, CDMA, and LTE and runs in iPhone, Android, Blackberry, Symbian and Windows Mobile devices. the operator can be notified in real-time of state of network. It is designed to test everything over IP and uses hardware for example Remote Test Unit 5 or RTU5 (Fig. 1) and software agents to actively test and monitor the performance and availability of services, such as VoLTE, SMS, email, VOIP, and CSFB. TEMS monitor master is managing with story boarder and dashboard that makes the test rapidly and easily. The software allows adhoc tests in distance located to solve IP problems.

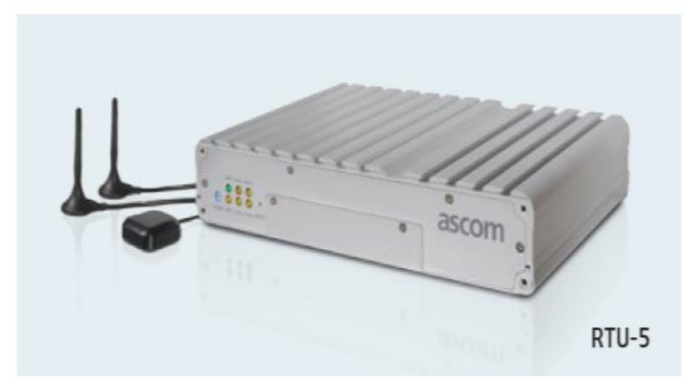

Fig.1. Ascom RTU 5.

The software is designed to measure wireless network anywhere that people go like restaurants, shopping malls, subways, trains, boats...so on. TEMS Pocket supports GSM/GPRS, EDGE, WCDMA, HSPA, CDMA, EV-DO, $\mathrm{Wi}-\mathrm{Fi}$, and LTE technologies. TEMS Pocket supports VoLTE testing and scanning with DRT4311B scanner or with PCTEL IBFLEX scanner. The software has service statistics view showing the outcome of executed service sessions, the history of cell usage and Rat.

The TEMS Capacity Manager supports [25] required data feeds from all Ericsson, Huawei, and ZTE OSSes for 2G, 3G (WCDMA), and 4G (LTE) networks. Unlike other products, It was designed to simplify the management of mobile network. With the use of Headroom reports the user is able to get a snapshot of the radio access network and where potential dimensioning issues may be arisen. TEMS Capacity Manager analyzes and detects the problems and the changes in network and proposes after the solution. Using it during the drive test, the engineer can easily identify the area network remediation, and efficiently prioritize where and when to expand the network. It supports multiple device types (including smartphones, modems, and PCIe MiniCards). It was designed for Benchmarking Drive test. It measures the same services, from the same location, at the same time. Different types of QoS tests can be executed in all types and generations of mobile radio technologies (GSM, GPRS, EDGE, CDMA2000, EV-DO, WCDMA, HSPA, and LTE). The TEMS Symphony GUI, Problem controller, lets users view and analyze data remotely. The Laptop is connected to the TEMS Symphony hardware called MTP-4 (figure.2), which is, in turn, connected to four phones. These test phones are placed in an isolation chamber MTP-4. Telephone mobile system Automatic [26] is designed to automate Drive testing. It supports GSM/GPRS, EDGE, WCDMA, HSDPA, HSPA+, Dual-Carrier HSPA, and LTE. The power of TEMS Automatic lies precisely in its capability to supervise and control probes remotely from a central location [27]. 


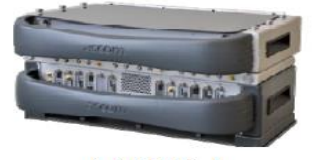

(a) MTP-4

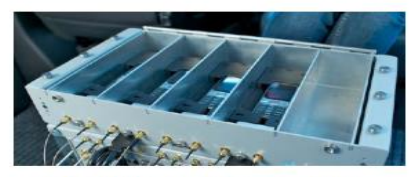

(b) MTP-4 with test phones
Fig. 2. Ascom MTP-4.

The feature gives to operators to simulate the user Smartphone' perceptive in such place: in subways, etc. the telephone mobile system discovery supports the following wireless technologies [28]: IS-95/ cdma 2000 1x, EVDO (Rev. 0/ Rev. A, B), GSM/GPRS/EDGE, WCDMA/ HSDPA/ HSUPA/ HSPA+, TD-SCDMA, WiMAX, LTE, and TD-LTE. TEMS Discovery automatically analyzes Drive test, user equipment, network OSS, crowd sourced, and application data, providing actionable intelligence via a Web-based management dashboard. The software accepted logfiles from others TEMS for analyses and post process. The item provides strong geolocation that allows to know where exactly the problem takes place. In term of features [29]. The proposed product supports LTE-A (Fig. 3), LTE (FDD and TDD), GSM, GPRS, EDGE, WCDMA, HSPA, HSPA+, TD-SCDMA, CDMA (IS-95 to EV-DO RevB).

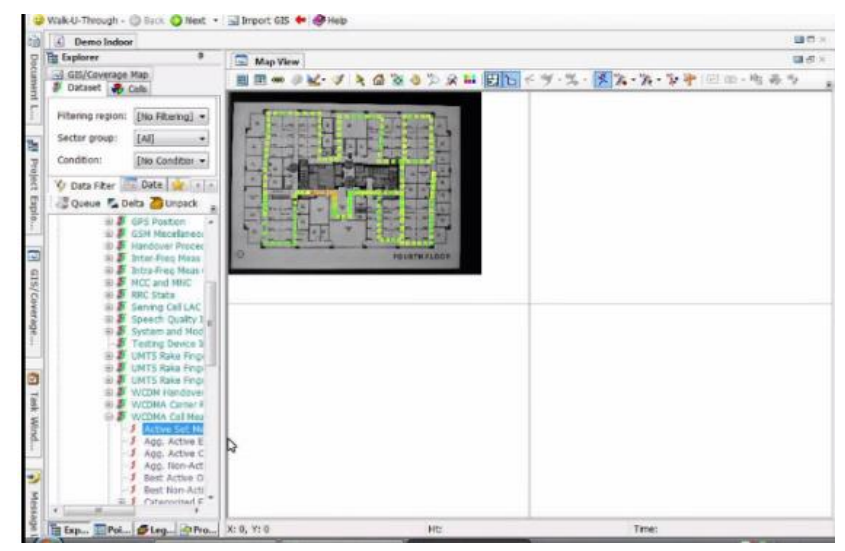

Fig. 3. Used TEMS discovery software Wimax.

Telephone mobile system facilitates service performance testing for voice, video telephony, FTP, HTTP, TCP, UDP, Ping, email, WAP, MMS, SMS, video streaming and VoIP. Voice quality is assessed with POLQA. Another TEMS Investigation features is scanning frequencies. It can be used with PCTEL SeeGull EXflex scanner. This devises scans LTE FDD, TD-LTE, WCDMA/HSPA(+), GSM, TDSCDMA, and CDMA/EV-DO frequencies from $300 \mathrm{MHz}$ to $3.8 \mathrm{GHz}$. Once installed the software, click Start Bar > Programs > Tems Product > Tems Investigation data Collection. This following window (Fig. 4) will appear.

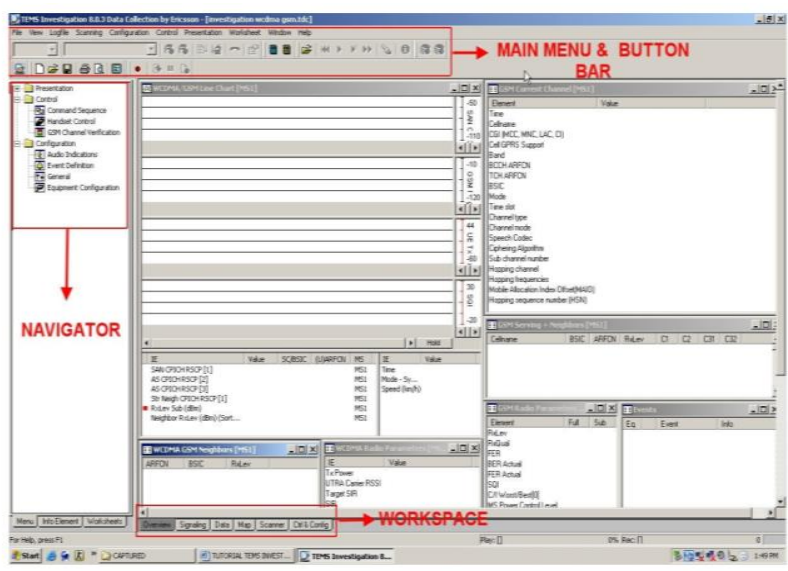

Fig. 4. TEMS Investigation screen print.

The presented model is divided into several sections:

- Main Menu and Button Bar: Here, we have logfile, presentation, scanning menus and button to connect all devices, button to start saving logfile, button to run a call, button to play an imported logfile and etc.

- Navigator : Presentation, Control, Configuration

- Workspace : We can switch between overview, signaling, date map, scanner, ctrl \& config page.

We connect the devices (GPS, mobile phone, scanner) to laptop via cable, and to TEMS software by pressing the corresponding button. By default, an assistant voice confirms the success or the failure of this operation. After that, we load the map where we do the Drive Test and add on it the cellfile and MapInfo Planning Tool (MIPT). Then, we start to configure the TEMS Software according types of Drive test and an aim of Drive Test. During tests, we need, at least, the following windows:

- Presentation (in main menu)>GSM> GSM Serving+ Neighbors: We have here BSIC, AFRCN, Rxlevel Full.. of serving cell and neighbouring cells.

- Presentation>GSM> GSM Line Chart : There are lines charts of RxLev, SQI and C/I worst of serving cell and bars of neighbouring cells.

- Presentation>GSM>GSM current channel : To seen all information about current channel

- Presentation $>$ GSM $>$ Radio Quality : This windows shows RxLev Sub.

- Presentation>Processing>GPS: We see here the latitude and longitude of each position.

The mobile is at the edge of two or more cells, there are no best server (cell) to keep a call. This situation causes what we call ping pong handover if there are more than two neighboring cells. As we can in (Fig. 5), value of RxLev stay low, $-85 \mathrm{dBm}$.

The undecoded $\mathrm{BCCH}$ or Broadcast Control Channel is 
logical channel used by base station to send some information about the network to mobile phone. Undecoded $\mathrm{BCCH}$ means the mobile phone can't decode the Base Station Identity Code (BSIC) of BTS in 5s. Many reasons caused that, one of them is channel interference and no dedicated spectrum for $\mathrm{BCCH}$.

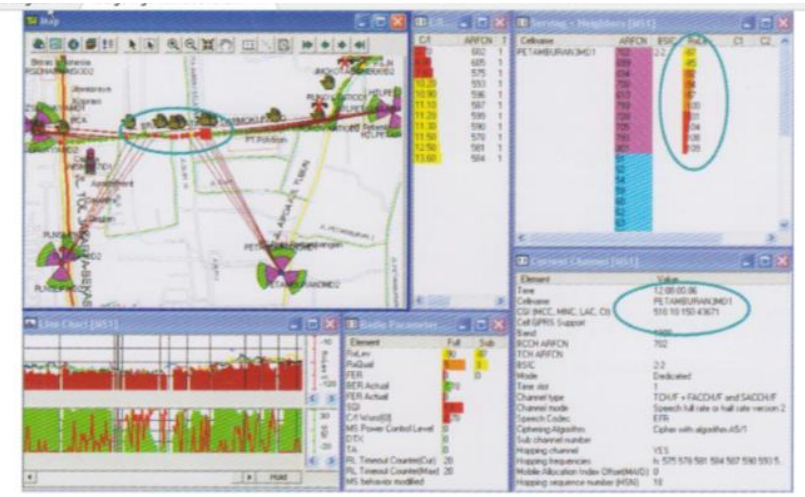

Fig. 5. No best dominant server.

Thus, the mobile phone can't connect to the network. In Drive Test, this situation is manifested on Serving+ Neighbors windows, for example in (Fig. 6)

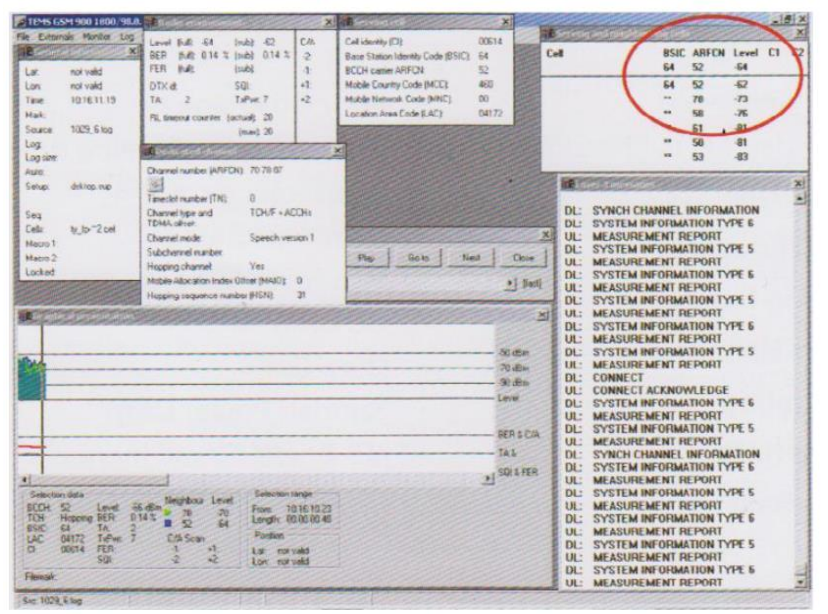

Fig. 6. Undecoded BCCH.

In UMTS, we need some windows:

- Presentation>WCDMA> Serving/ Active Set+ Neighbors : Here, we have ceel I.D, cell name, scrambling code of serving cell and neighbouring cells.

- Presentation>WCDMA>Radio Parameter: On this window, we see the WCDMA parameters.

- Presentation>WCDMA> Line Chart WCDMA : Like in GSM, this window shows

Common Channel Pilot Channel ( or CPICH ) RSCP, UE Tx power, and CPICH Ec/No.

- Presentation>WCDMA> GSM neighbors : We need this window about $2 \mathrm{G}$ neighbors for $2 \mathrm{G}-3 \mathrm{G}$ interference and handover.
The Pilot pollution is a situation in which a mobile station receives several pilot signals with strong reception levels, but none of them is dominant enough that the mobile can track it. Since all Node B in the network use a same frequency, this situation causes interference to the mobile. To avoid it, planner network allows only 3 (in general) pilot signals in range $5 \mathrm{~dB}$ form the strongest or serving pilot. During Drive Test (Fig.7), there is a pilot pollution if the number of Node B in active set exceeds 3 .

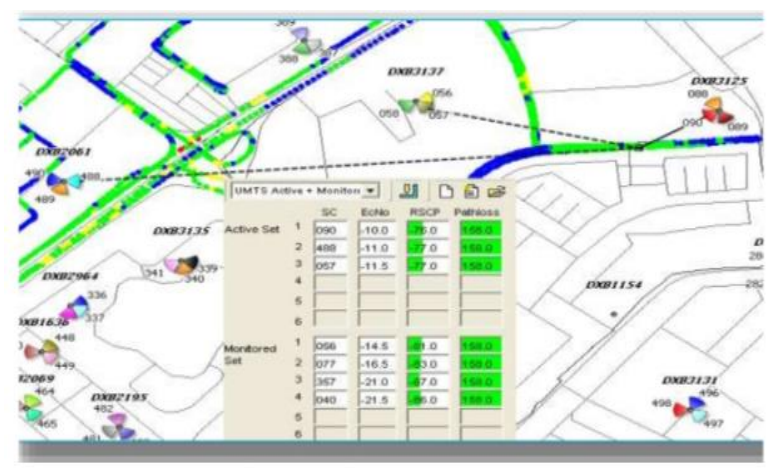

Fig. 7. Pilot Pollution.

\section{POOR COVERAGE STUDY BASED ON MISSING NEIGHBORS}

The mentioned problem is identified. Tx Power is very low that indicates increasing error of received bits. In Serving/ Active Set+ Neighbors window, the Detected Neighbor (DN) has the best Ec/Io and RSCP is not added to Active Set (AS) due missing neighbors (figure.8)

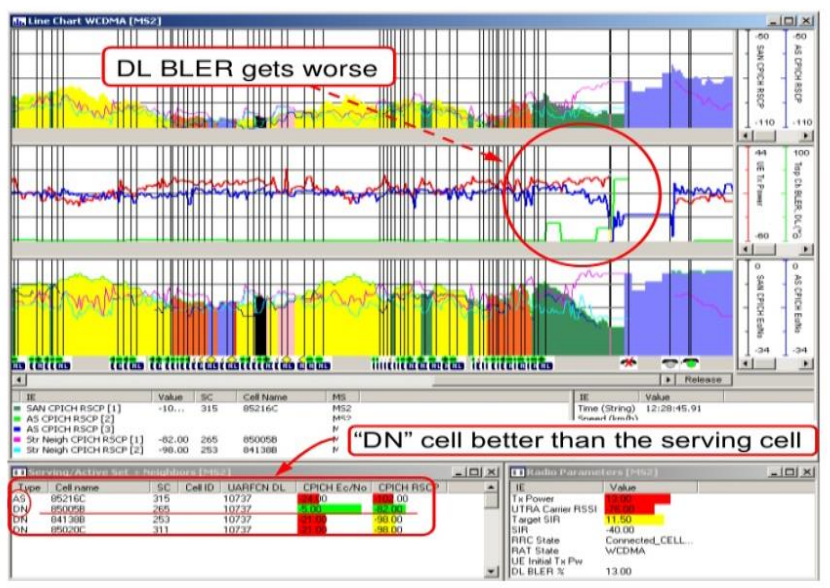

Fig. 8. Poor coverage.

The Ascom is a software for Drive test according how the measurement takes place and will be managed. TEMS Investigation is one of them provides the best features for taking and analyzing the logfile. I have presented a few examples of problem detected by TEMS investigation, but 
the list is long and some of problems are due to interference in mobile radio.

The spectrum is a rare resource and many organizations on international and national level like Frequencies National Agency in Tunisia, manage the division of spectrum. One of Their roles is to distribute a specific range of frequency to telecommunication societies in order to avoid the interference problem. In mobile radio, its interminable fight, interference problem exists both in mobile phone and in base station. As you can see, major problems seen above caused by or cause interference. In this chapter, we concentrate on interference in general in first section. After, we will see what are and how mobile system combat it. As known, in radio telecommunication, the interference is defined as a sum of all signal adding to useful signal. It's an artificial noise and it's generated by another device that the useful signal is not designated to. It affects the signal quality and reduces the performance of wireless. There are many solutions hard and soft against the different types of interference. If we look for sources of interference, the major cause of interference in field can be another mobile in the same cell, a call in progress in the neighboring cell, other base stations operating on the same frequency, and any non-cellular system which leaks energy into the cellular frequency band. The effects of Interference is not reciprocal in the uplink and downlink direction. The mobile stations and base stations are affected by different interference situation because they are located in different electronic environment. Both are exposed to following problems such interference is a major limiting factor in the performance of cellular systems, the degradation of signal quality, introduces bit errors in the received signal, and bit errors are partly recoverable by means of channel coding and error correction mechanisms. The interference in Drive test phenomenon is mainly characterized by a good reception of signal with high number of bits decoded incorrectly. It means we have a high Rxlevel but bad value of Rxqual or BER as you can see in the figure.9, Rxlevel=$60 \mathrm{~dB}$ and $\mathrm{BER}=10$.

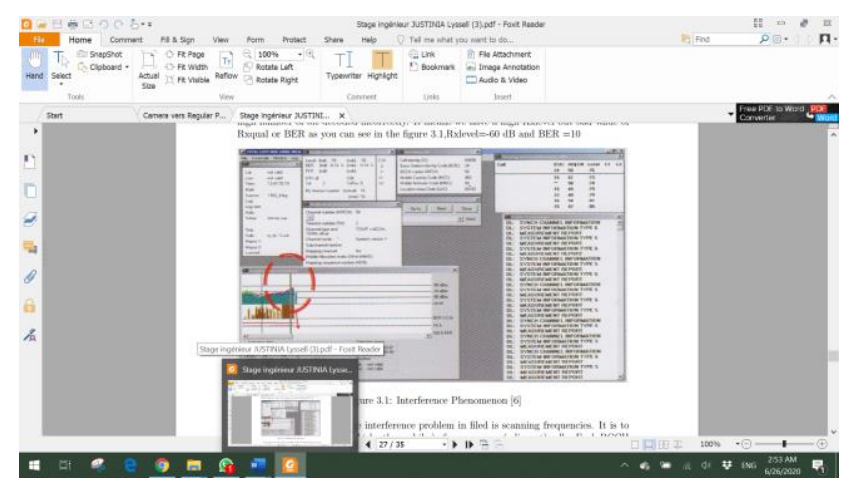

Fig. 9. Interference Phenomenon [6].
Another way to find some interference problem in filed is scanning frequencies. It is to measure $\mathrm{BCCH}$ levels (caught by the mobile) of one or more (adjacent) cells. Each $\mathrm{BCCH}$ has its frequency designed by a Absolute RadioFrequency Channel Number (ARFCN for GSM and UARFCN for UMTS) in its specific area.

Here, we have scanned three frequencies: $928.4 \mathrm{MHz}(-$ $70 \mathrm{dBm}), 1836.8 \mathrm{MHz}(-100 \mathrm{dBm})$ and $1837 \mathrm{MHz}(-90 \mathrm{dBm})$ respectively corresponding to ARFCN: 991,671,670. Depending on these Rxlevel values of cells we can define some types of interference. In practice, we have different types of Interference:

-Adjacent-Channel Interference (ACI), this interference is a result of imperfect receiver filter so there is remain of signal for adjacent channel. Nonlinearity one frequency radiates into another frequency's spectrum cause also adjacent channel interference. This also happens if the Mobile is far away from its transmitter and near another using adjacent channel. In order to avoid this situation, proper radio frequency planning with appropriate guard bands between the carriers need to be adopted. The carrierto-adjacent ratio is defined as the signal-strength ratio between a serving carrier and an adjacent carrier. It is calculated according to the following formula:

$\mathrm{C}=\mathrm{A}(\mathrm{dB})=$ Serving signal strength $(\mathrm{dBm})$ \{ Adjacent signal strength $(\mathrm{dBm})$. for the intersymbol interference (ISI), it happens when the previous symbols transmitted affect like a noise to the decision of current symbol. The ISI is usually caused by the multipath of wireless signal. There are many copies of signal arrived to the receiver result of reflection, refraction and atmospheric effects. The second cause of ISI is Bandlimited channels. Filtering signal in such as a channel changes a shape of pulse within first symbol and it's spread over the symbol periods. The GSM system uses constant envelope GMSK modulation, which is continuous phase modulation, robust against signal fade as well as interference and have good spectral efficiency. At the output of GMSK modulator, where fc is carrier frequency. bk is 1 if the squares of pulse corresponding to the information aI at $[(\mathrm{k}-1) \mathrm{T},(\mathrm{k}+1) \mathrm{T}]$ and aQ at $[\mathrm{kT},(\mathrm{K}+2) \mathrm{T}]$ are same, and -1 if else. $\theta \mathrm{k}$ is 1 ifa $=1$, and $\pi$ otherwise. The parameter of GMSK filter-bandwidth $*$ bit interval product $(\mathrm{B} * \mathrm{~T})$ is fixed at 0.3 to avoid the interference and the problem of band width. In order to avoid intersymbol interference, GSM use also tailing bits (TB) at the beginning and at the end of bursts to reset he memory of the Viterbi channel Equalizer (VE) which controls the intersymbol interference. Structure of timeslot in GSM [13] UMTS standard use spreading codes to make different between users. Each user has its unique spreading code, which is multiplied by data to obtain the Chips. 
For the Code Tree spreading factor and to get the actual rate bit of CDMA in UMTS, it is simply divide the chips rate by the SF. All codes having a same SF and all codes having a different mother code are orthogonal between them. Scrambling code is random code to avoid inter-base interference.

The co-site interference and inter-operator interference Co-site is a site with different mobile systems. This situation creates inevitably interference. For example, UMTS 900 and GSM 900, bandwidth of 4.2 MHz is used in UMTS to ensure the capability and quality of GSM. Geographical isolation". It means the UMTS and the GSM using same band frequency work in different zones. Interoperator interference occurs when two operators using adjacent frequencies in same site. Interfering BTS A transmits some power signal to interfered operator B due to an imperfect transmit filter. That is considered as Adjacent Channel Leakage Ratio or ACLR. The Adjacent Channel Selectivity or ACS happens when operator B has a bad receiver filter and can't eliminate signal in adjacent frequency.

The crossed feeders or feeder cable is a cable used to transfer radio frequency energy from one point to another antenna. When feeder of two or more cells are accidentally connected, it's crossed feeders. It degrades the performance of network. There are there types of crossed feeders such crossed transmit feeders, crossed received feeder, and crossed transmit and receive feeders: for crossed transmit feeders is the most damaging. Two cells or more swap their $\mathrm{BCCH}$ and $\mathrm{TCH}$ frequencies sectors. This creates an important interference to the network and handover is rarely successful. The map where occurs Crossed Transmit Feeders, the cell site has 3 sectors: $\mathrm{A}$ and its $\mathrm{BCCH}$ frequency is in red, $\mathrm{B}$ represented by green and $\mathrm{C}$ represented by pink. After scanning the field, we get this image. A and B are swapped due to crossed transmit feeders. This situation changes the site planning and develop, as an example, co-channel interference. To detect the crossed received feeders, we need to analyze the static of up-link signal strength (figure.10), handover success rate and others. By using Drive test, it's difficult task because crossed received feeders do not arise a specific problem like in the crossed transmit feeders. About crossed transmit and receive feeders, as the name implies, it's mix between crossed transmit and receive feeders.

\section{CONCLUSION}

We analyzed several phenomena in adjacent site operators, and we conclude that operators should coordinate their used frequencies, this is important to avoid BTS interfering phenomena. The problem can be emerged if BTSs of the same operator are set with wrong parameters. GSM radio interferes with UMTS radio when an adequate isolation is not provided. Fortunately, electronic and or mechanical, numerical or analogical systems used to reduce the problem. Network problems can be arisen from the core network to transceiver and from transceiver to the mobile phone's subscriber. Troubles and malfunctions troubleshooted by Drive test. In fact, TEMS investigation, Drive test software generates a file called logfile which contains network parameters and signal parameters. That's why it allows analyzing the problem in the field or post Drive Test and proceed to enhancement. Otherwise, if everything seems right on this part, to affirm the trouble is in the first part of network. Interference, one of the major radio communication problems, augments level with arrival of new technologies, construction of buildings, increasing number of subscribers. To avoid it in cell coverage is a real network optimization for expert. Reducing the signal strength from transceiver arises another problem like no dominant server, sudden decrease on signal level and increasing this signal level causes for instance Pilot Pollution.

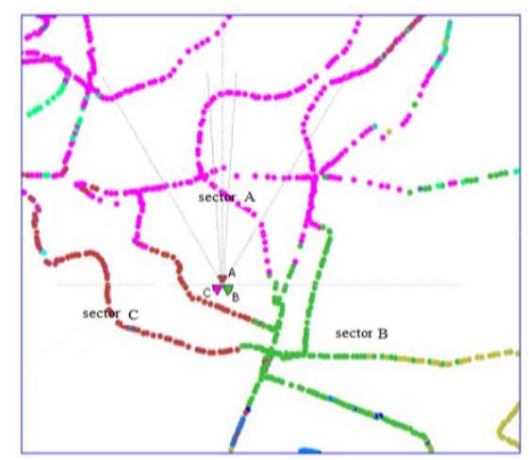

Fig. 10. Crossed Transmit Feeders.

\section{Acknowledgement}

The Author would like to acknowledge the financial support of this work by grants from the Taif University T.U, Kurma University College, Kingdom of Saudi Arabia.

\section{REFERENCES}

[1] Q. Yu, L. Yan, S. Lee, "Loop synchronous polarization scrambling technique for simulating polarization effects using recirculating fiber loops," Journal of Lightwave Technology, vol.21, pp.1593-1600, Jul. 2003.

[2] N.S. Murthy and P. Srikanth Gautham, "Adaptive Equalizers in Smart Antenna Systems," IETE Journal of Education,vol.50, pp. 111-117, Sep. 2009.

[3] A. H. Levy and A. S. Willsky, "An efficient maximum entropy technique for 2-D isotropic random fields," IEEE Journals \& Magazines, vol. 2, pp.741-744, Apr. 
1988.

[4] P. Seong Chuah, C. Zhenzhong and Y. C. Peng Tan, "Energy Minimization for Wireless Video Transmissions With Deadline and Reliability Constraints," IEEE Transactions on Circuits and Systems for Video Technology, vol. 23, pp.467- 481, Mar. 2013.

[5] M. Vesperini, F. Breon and D. Tanre,"Atmospheric water vapor content from space borne POLDER measurements," IEEE Transactions on Geoscience and Remote Sensing, vol. 37, pp.1613-1619,Feb. 1999.

[6] A.Verma, N. Srivastava and B. K. Kanaujia, "Analysis of Gunn Loaded Rectangular Microstrip Antenna," IETE Journal of Education, vol. 53, pp.80-87, Jul. 2012.

[7] K. L. Rao Member, "A Simplified Approach to the Antenna Problem," IETE Journal of Education, vol. 23, pp. 7-8, Jan. 1982.

[8] Natalia C., Estefania A., Vicente B.,"THOMAS: An agent platform for supporting normative multi agent systems," Journal of Logic and Computation, vol. 23, no. 2, DOI: 10.1093/logcom/exr025, pp. 309-333, 2013,

[9] Frank L. Lewis, Bing Cui, Tiedong Ma, Yongduan Song, Chunhui Zhao, "Heterogeneous MultiAgent Systems:Reduced-Order Synch- ronization and Geometry," IEEE Transactions on Automatic Control, vol. 61, no. 5, pp. 1391-1396, DOI: 10.1109/TAC.2015. 2471716, 2015.

[10] Z. Guessoum, "Adaptive agents and multiagent systems," IEEE Distributed Systems, vol. 5, no. 7, DOI: 10.1109/MDSO.2004.10, 2004.

[11] Hao Zhang, Gang Feng, Huaicheng Yan, Qijun Chen, "Distributed self-triggered control for consensus of multi-agent systems," IEEE/CAA Journal of Automatica Sinica, vol. 1, no. 1, pp.40-45, DOI: 10.1109/ JAS.2014.7004618, 2014.

[12] Meng Guo, Michael M. Zavlanos, Dimos V. Dimarogonas, "Controlling the Relative Agent Motion in Multi-Agent Formation Stabilization," IEEE Transactions on Automatic Control, vol. 59, no. 3, pp.820-826, DOI: 10.1109/ TAC.2281480, 2014.

[13] P K Singhal, Pankaj Singh Tomar and Neha Verma, "Topologies and Applications of Meander Line Antenna," IETE Journal of Education, vol. 48, pp.121-131, Jul. 2007.

[14] P. Gebhard, "A Layered Model of Affect," in Proceedings of the Fourth International Joint Conference on Autonomous Agents and Multiagent Systems (AAMAS '05), pp. 29-36, New York, NY, USA, 2005.

[15] Q. Yu, L.S. Yan, S. Lee, Y. Xie and A. Khilla and I. Wolff, "Field Theory Treatment of H-Plane Waveguide
Junction with Triangular Ferrite Post," IEEE Transactions on Microwave Theory and Techniques, vol. 41, pp. 274-281, Feb. 1993.

[16] K. Ghamen and A. Caplier, "Estimation of facial expression intensity based on the belief theory," in Proceedings of Funchal, Portugal, pp. 59-60, Jan. 2008.

[17] M. Dammak, A. Wali and A.M. Alimi, "Video summarization using viewer affective feedback," In Proceedings of 13th International Conference on Hybrid Intelligent Systems (HIS), pp. 279-284, Dec 2013.

[18] Tianyi Xiong, Zhiqiang Pu, Jianqiang Yi, Xinlong Tao, "Fixed-time observer based adaptive neural network time-varying formation tracking control for multi-agent systems via minimal learning parameter approach," IET Control Theory \& Applications, vol. 14, no. 9, pp. $1147-1157$, DOI: 10.1049/iet-cta.2019.0309, June 2020 ,

[19] Meng-Lin Li and Shaofei Chen, “ Adaptive Learning: A New Decentralized Reinforcement Learning Approach for Cooperative Multiagent Systems," IEEE Control Systems Letters, vol. 4, no. 4, pp. 898 - 903, DOI: 10.1109/LCSYS. 2020.2995756hen, 2020.

[20] Xiaoyuan Luo, Xianluo Li, Xiaolei Li, Jing Yan, Xinping Guan, "Globally Stable Formation Control of Nonholonomic Multiagent Systems With Bearing-Only Measurement," IEEE Systems Journal, vol.14, no. 2, pp. 2901-2912, DOI: 10.1109/JSYST.2019.2935162, August 2019.

[21] Shangfei Zheng and Hong Liu, "Improved MultiAgent Deep Deterministic Policy Gradient for Path Planning-Based Crowd Simulation," IEEE Access, vol. 7, pp. 147755 - 147770, DOI: 10.1109/ACCESS.2019.2946659, October 2019.

[22] Dazhuan Xu, Dan Chen, Ruidan Zhang, "Performance and optimisation of agent compression and forwarding system for correlated sources," IET Control Theory \& Applications, vol. 13, no. 16, pp. 2523-2532, DOI: 10.1049/iet-com.2018.5704, Oct. 2019.

[23] W. Kempton and A. Dhanju, "Electric vehicles with V2G: storage for large-scale wind power," Windtech International, vol. 2, no. 2, pp. 18-21, 2006.

[24] M. D. Galus, and G. Andersson, "Balancing Renewable Energy Sources using Vehicle to Grid Services controlled by MPC in a Metropolitan Area Distribution Network," in Proceedings of CIGRE ELECTRA, pp. 1-18, 2012.

[25] P. Mesaric and S. Krajar, "Home demand side management integrated with electric vehiclesand renewable energy sources," Energy and Buildings, vol. 108, pp. 1-9, 2015.

[26] M. D. Galus, M. G. Vayá, T. Krause, and G. 
Andersson, "The role of electric vehicles in the smart grid," Wiley Interscience Interdisciplinary Reviews of WIREs, Energy andEnvironment (WENE), vol. 2, no. 4, pp. 384-400, July/Aug. 2013.

[27] W. Kempton and J. Tomic, "Vehicle-to-grid power fundamentals: calculating capacity and net revenue," Journal of Power Sources, vol. 144, no. 1, pp. 268-279, 2005.

[28] J. Suk-Yee, "Domestic electric vehicle market showing rapid growth," Business Korea, April 15, 2015/10126-governmental-support-domestic-electricvehicle-market-showing-rapid-growth. Retrieved on 13-06-2016.

[29] S. Boyd and L. Vandenberghe, Convex Optimisation, Cambridge University Press, New York, United States of America, 7th Edition, pp. 1-2, 2009.

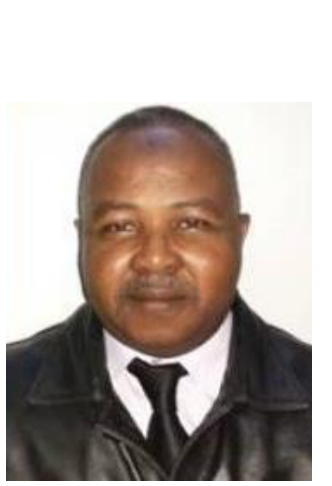

Author

Chafaa HAMROUNI was honored as IEEE Senior member 2008, Chair IEEE AESS R8 Society from 20102014 and IEEE CEDA R8 Society from 2015-2018. Currently he teaches in the Kingdom of Saudi Arabia, at the University of Taif (TU), Khurma University College (KUC), Department of Computer Sciences. He is a Ph. D. Engineer in Computer Eng. He is expert in Wireless Network and Networking in Telecom Studies and Research Center for 15years. Research topic Information Technologies, Intelligence Technologies and Pico Satellites development. He received his MS and PhD degrees in the Department of Research Groups on Intelligent Machines from the Sfax University of Sfax., Tunisia, in 2008 and 2013, respectively. In 2013, he joined the Department of Robots and Computer Engineer for pursuing his $\mathrm{Ph}$. D. degree at Wurzburg University. His research interests include Small Satellites Technologies, IT, Metworks, Alghorithms. Dr. Chafaa Hamrouni received his HDR Diploma in 2019. He conducted several international projects with Turkey, South Africa and Germany. 
New Trend Proposal in Optimization Techniques Application for Mobile Network, Analysis and Signal Processing 